\title{
Editorial
}

\section{Robotics - Inspired from Nature}

\section{Huosheng Hu}

School of Computer Science \& Electronic Engineering, University of Essex, Colchester, CO4 3SQ, UK; E-Mail: hhu@essex.ac.uk; Tel.: +44-1206-872297; Fax: +44-1206-872900

Received: 16 April 2012 / Accepted: 17 April 2012 / Published: 24 April 2012

It is my great pleasure to welcome you to a new open access journal, Robotics, which is dedicated to both the foundations of artificial intelligence, bio-mechanics, mechatronics and control theories, and the real-world applications of robotic perception, cognition and actions. This includes the innovative scientific trends, and discovery resulting from solving new challenges in the field of robotics. Its open access and rapid dissemination are the unique features separating this journal from all existing journals dedicated to robotics.

Over the last few decades there has been an exponential growth in intelligent machines and robots, which has led to the invention of exciting new products that are now in daily use such as dishwashers, washing machines, auto cameras, computers, mobile phones, robotic pets, service robots, etc. However, the operation of all these machines or robots does not really require them to "think" as they are simply programmed to perform a series of predefined tasks repetitively. If anything interferes with the pre-programmed tasks, they shall malfunction, since they are not able to sense the unexpected interference and think out a solution creatively. It remains a great challenge today for us to build truly intelligent machines and robots that can "think" like we do.

Inspired from nature, engineers and scientists have been trying very hard to capture the essence of human intelligence in our machines and robots to allow them to function well in the real world. Like human beings, intelligent machines and robots should be able to sense their environments, reason and make decisions, and respond to tasks and unexpected events quickly. In other words, they need a fast and multi-layer connection from perception to action autonomously without human intervention. This is a very challenging and ambitious task since the robot or machine intelligence must cope with various noises, uncertainty, and dynamic changes in the real world.

As a subject, robotics is a broad, interdisciplinary one that involves many different technologies such as artificial intelligence, sensor data fusion, embedded computing, perception, recognition, navigation, communication, planning and learning. For each individual robot, the "thinking" process should take place at many different levels. At its lowest level, "thinking" needs to be fast to response quickly to unexpected events, e.g. keeping balance or avoiding 
obstacles. At higher levels, "thinking" enables the robots to deal with a dynamic and uncertain world, e.g. exploring a new space and finding a safe way to go. In contrast, "thinking" should exhibit adaptation and learning capability at its highest level, e.g. learning from imitation and experience as humans do. Moreover, a team of robots and intelligent machines that are operated together should be capable of communication and coordination to achieve the common goal cooperatively.

To have a machine or robot that is completely autonomous or truly intelligent was only a dream yesterday, which was reflected by some science fiction books and films such as "I Robot" and "Dr. Who". It remains a dream today since most of the autonomous robots and intelligent machines currently being built do not function well in the real world. This is mainly attributed to our incomplete understanding of the processes of perception, cognition and reasoning in humans, the limitations of available scientific methods, and the incompatibility of today's computer and sensor technologies. However, our dream will become reality with future research efforts. This is the motivation for the creation of this new journal.

The major goal of this new open access journal, Robotics, is the publication of new research results on robots and intelligent machines, as well as their successful real-world applications. The aim is to provide an international forum to report current developments from interdisciplinary theoretical studies, computational algorithm development, and applications of robotic systems. Robotics particularly welcomes those emerging methodologies and techniques which bridge theoretical studies and applications in all robotic systems, including novel quantitative engineering and science studies.

Robotics will provide very rapid dissemination of novel scientific findings. All articles will be peer-reviewed to assess whether they are scientifically sound and properly written. If work is well conducted and properly documented, then it is a contribution to the field and only the future can determine its true impact or significance. We want to accelerate the progress from discovery to dissemination. Review and a decision on publication will be rapid. We believe the time that scientists have is precious and is better spent on research and discovery rather than on rewriting articles.

Robotics has a superb and diverse editorial team that is committed to making the journal a success. Our aim is to promote research by reducing the time that publishing results takes out of busy research schedules. The journal will be indexed timely in PubMed and other standard search and indexing services. It will benefit from the Publisher's experience of more than ten years with a range of successful online journals and the crisp web interface and submission procedure. The launch of a new journal is an exciting moment and we encourage you to submit your articles to Robotics now.

The 21 st century is the robotics century and intelligent robots will change our lifestyle forever. Let us face the challenge and work together toward the realization of intelligent machines and robots step by step. It is great fun to create intelligent machines around us and imagine practical applications of intelligent robots to improve our quality of life. Robotics is now ready to serve you in a long journey towards such a goal.

(C) 2012 by the authors; licensee MDPI, Basel, Switzerland. This article is an open access article distributed under the terms and conditions of the Creative Commons Attribution license (http://creativecommons.org/licenses/by/3.0/). 